\title{
Scoping Biocatalyst Performance using Reaction Trajectory Analysis
}

Nordblad, Mathias; Dias Gomes, Mafalda; Meissner, Murray Peter; Ramesh, Hemalata; Woodley, John

Published in:

Organic Process Research and Development

Link to article, DOI:

10.1021/acs.oprd.8b00119

Publication date:

2018

Document Version

Peer reviewed version

Link back to DTU Orbit

Citation $(A P A)$ :

Nordblad, M., Dias Gomes, M., Meissner, M. P., Ramesh, H., \& Woodley, J. (2018). Scoping Biocatalyst Performance using Reaction Trajectory Analysis. Organic Process Research and Development, 22(9), 11011114. https://doi.org/10.1021/acs.oprd.8b00119

\section{General rights}

Copyright and moral rights for the publications made accessible in the public portal are retained by the authors and/or other copyright owners and it is a condition of accessing publications that users recognise and abide by the legal requirements associated with these rights.

- Users may download and print one copy of any publication from the public portal for the purpose of private study or research.

- You may not further distribute the material or use it for any profit-making activity or commercial gain

- You may freely distribute the URL identifying the publication in the public portal 


\title{
Scoping Biocatalyst Performance using Reaction
}

\section{Trajectory Analysis}

Mathias Nordblad, Mafalda Dias Gomes, ${ }^{+}$Murray P. Meissner, ${ }^{+}$Hemalata Ramesh, John M.

\author{
Woodley*
}

Department of Chemical and Biochemical Engineering, Technical University of Denmark, Building 229, Søltofts Plads, DK-2800 Kgs. Lyngby, Denmark 
TOC FIGURE

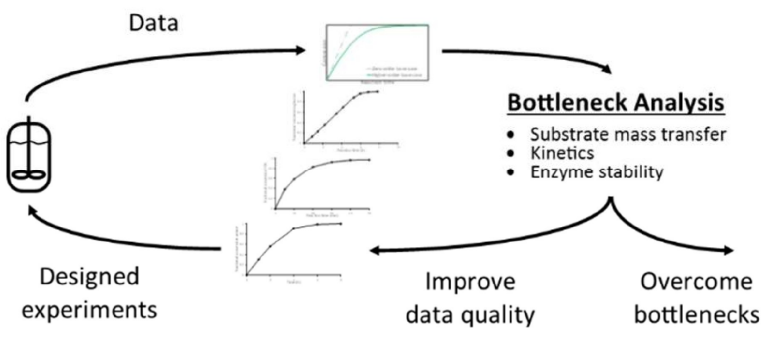


KEYWORDS. Biocatalysis; Process Development; Kinetic Characterization; Bottleneck Identification

\begin{abstract}
Process development for biocatalytic reactions is a complex task due to the required interaction of several different scientific disciplines. Additionally, there is a lack of standardized procedures for guiding development and for identifying the major process limitations in these systems. This work seeks to address this problem by providing a methodology based on a simple, systematic series of experiments. Application of the methodology helps identify the major bottleneck for process implementation, whether it be enzyme activity, enzyme stability, or substrate mass transfer. In addition, the underlying mechanism behind these limitations can also be inferred. The methodology is illustrated using a simulated reaction system and is also applied to three experimental case studies. This methodology provides a set of simple experiments that may be performed at an early stage of biocatalytic process development to guide effective improvement strategies, whether they be via protein engineering or reaction engineering. Ultimately, this should afford faster and more efficient implementation of biocatalysts in industrial processes.
\end{abstract}

\title{
Introduction
}

The use of conventional synthetic methods to produce interesting and valuable chemical products is today challenged by the desire to use sustainable feedstocks (cheap and readily available raw materials from renewable sources) and processes that necessitate highly selective 
catalysis. A key technology required to implement these more sustainable production routes is biocatalysis (using enzymatic catalysts or microbial cells containing enzymes, produced by fermentation). ${ }^{1}$ Biocatalytic processes come with many benefits, including the potential to catalyze reactions at very high selectivity under mild conditions. Despite the strong motivation to implement such technology in industry, only a few hundred processes have been commercialized to date. ${ }^{2}$ While many processes have been developed in the pharmaceutical industry ${ }^{3-13}$, applications can also be found in the food, flavors and fragrance industry and even in the bulk chemicals sector. ${ }^{14,1}$ The dominating challenge in all these cases has been, and continues to be, the complexity of the design task. ${ }^{15}$

The flexible nature of enzymes have allowed them to evolve over time to fit perfectly for their roles in Nature; a fit that typically requires a balance between enzyme activity and stability as well as substrate affinity and selectivity. In many cases, the intended substrate/product and/or desired reaction conditions for industrial implementation of a reaction of commercial interest will be different from that which can be found in Nature. For example in the pharmaceutical industry new therapeutic targets are sought, which by definition have not been seen in Nature. ${ }^{16,17}$ In all cases the conditions required for commercialization (such as high productivity and product concentration) are not the usual conditions for enzyme catalysis. ${ }^{18,19}$ Molecular biology provides tools allowing the possibility to swap the constituent amino acids and enables alteration of an enzyme's properties, a technique referred to as protein engineering. ${ }^{17}$ The modification of an enzyme by protein engineering can be beneficial towards improving enzyme activity $^{20}$, and stability ${ }^{21}$ as well as substrate affinity/selectivity ${ }^{22} \cdot{ }^{16,23}$ However, the modifications required are specific for each target, and an improvement of one property may even come at the expense of one or more of the others. Protein engineering is one of the most 
valuable features of biocatalysis, but from the design perspective it represents an extra degree of freedom that needs to be optimized. Further, a recent paper emphasized the need for much greater speed to be achieved in the protein engineering methods themselves. ${ }^{24}$ Hence, establishing a specific protein engineering target for process improvement is invaluable at an early stage of the design process because it will reduce the overall development time required to engineer a catalyst for industrial implementation.

Beyond the work on engineering the enzymes themselves, process development has to date been perceived to take too long. This is in part a consequence of the extra degree of freedom which comes from the ability to alter the biocatalyst, but also because of the complicated interacting phenomena (between the physicochemical components of the reaction system and the catalyst) that dictate the characteristics of a particular biocatalyst.

The complete development of a new process based on biocatalysis requires the involvement of a broad range of scientific and engineering disciplines. Starting with the initial identification of catalytic activity for a desired reaction, the candidate enzyme will likely require modification to improve catalytic performance; will need to be transferred into a host for more efficient production; formulated in a way that is suited for process implementation (e.g. through immobilization); characterized to identify reaction performance under select conditions; and ultimately, when performance is deemed economically feasible for implementation, a process will need to be designed around the reaction. Since each of these aspects play a role in determining the ultimate performance of the process, the development process is typically nonlinear. In order to accelerate the entire course of progression, standardized methods and tools such as for enzyme production, immobilization, and performance evaluation would be highly desirable. $^{25}$ 
The development of a biocatalytic process must be supported by empirical experiments to assess the improvements achieved after applying available engineering tools. For certain types of assessment there are well-established methods to provide the required information. Examples of such experiments include measuring reaction progress after a certain reaction time (single-point measurements), or so-called 'initial rate measurements' ${ }^{26}$ The output of such studies include kinetic parameters of the enzyme rate law, as well as stability, under different conditions. ${ }^{27}$ Although laborious, these types of experiments are used extensively today in scientific investigations to characterize biocatalysts.

In the context of process development, the above-mentioned methods are also useful to inform feasibility assessments and for optimizing reaction conditions for a given system. However, at an early stage of process development there is also a need to establish the main limitation, or bottleneck, in the reaction system of interest, to guide further catalyst (protein engineering) and/or process development. While a simple measure of reaction progress at a specific time may be sufficient to determine that an improvement is needed, it cannot distinguish what improvement is required. On the other hand, a detailed investigation to fully characterize a new reaction system is excessive at an early stage of development. Instead, a method is needed that also offers qualitative information regarding the reaction system (such as the bottleneck under the relevant conditions). Unfortunately, while the experimental approaches to quantifying performance, kinetics etc. are well established, there is far less consensus on how bottleneck analysis should be approached. Instead, prior knowledge and ad hoc experimentation is typically used to guide development efforts.

In an attempt to address this challenge towards the wider implementation of biocatalysis we present here a methodology to guide scientists and engineers through the early process 
evaluation stage. The approach is inspired by the prior works of Blackmond ${ }^{28}$ and Duggleby ${ }^{29}$, where high quality/high frequency measurements were used to determine dynamic changes in catalytic activity over the course of a reaction and thus elucidate catalytic mechanisms. However, the approach we have taken goes a step further relying on standardized plots of reaction progress against reaction time, similar to an early approach by Selwyn. ${ }^{30}$ Building on Selwyn's work (which is a method to identify biocatalyst inactivation), we have added experiments to distinguish between limitations in mass transfer, biocatalyst kinetics and stability, as well as the underlying mechanisms behind these phenomena. This approach enables the use of a few, relatively simple, laboratory experiments to guide and inform not only protein engineering and process design efforts, but ultimately to assess the feasibility of scaling such processes for industrial implementation. This is particularly important in biocatalysis since screening at a laboratory scale, unless specifically targeted, rarely distinguishes multiple-traits under industrially-relevant conditions. Hence, we argue that using such a methodology at an early stage should enable strategic decisions to be made prior to significant research investment, thereby reducing costs and increasing confidence in the decisions made. Lastly, it ought to be mentioned that this method only pertains to experimentally characterizing available enzymes towards a process performance in both a qualitative and quantitative manner and does not address the subsequent protein engineering or reaction optimization efforts.

The methodology presented in this study is based on the graphical analysis of reaction trajectories. The methodology is illustrated through simulated reactions based on relevant models for enzyme kinetics and stability as well as mass transfer limitations, with theory provided to explain the underlying phenomena. Subsequently, it has been applied to experimental data from three separate case studies, each presented in the Results and Discussion section. 


\section{Theoretical Basis}

The progression of a reaction - how the conversion of substrate to product occurs over time is dependent on reaction kinetics which in turn are dictated by the composition of the reaction mixture at any given time, as well as any effects that accumulate over time. In particular, the rate of a biocatalytic reaction is determined by the reaction composition as dictated by enzyme kinetics (and any mass transfer limitations), but can also be affected by loss of catalytic activity that occurs over the course of the reaction.

Different experimental approaches will lead to different results, and emphasize different aspects of the performance of a reaction system. For example, single-point experiments aggregate all effects into a single number - catalyst kinetics and mass transfer limitations, both of which may change with reaction composition, and any loss of catalyst activity over the course of the reaction due to inactivation. Initial rate experiments on the other hand can isolate effects on reaction kinetics, but cannot account for the accumulated effect of reaction time. Fully characterizing the impact of changing reaction composition on reaction performance over the entire reaction requires extensive experimentation with the initial rate approach. In an assessment of the full reaction progression, all effects are aggregated in each individual data point (just as for single point experiments). However, the way the effects interact to define the reaction trajectory (the shape of the reaction curve) is distinctive, though not necessarily unique, for a given dominating phenomena in a reaction. The accuracy in determining the shape of a reaction trajectory is enhanced through higher frequency measurements, such as those which direct, online, analytical techniques can provide. ${ }^{31}$

THE REACTION PLOT. In any kinetic study involving biocatalysis, it is common to see at least one plot of the reaction course. ${ }^{32}$ The reaction plot can be used to illustrate how the 
performance changes over the course of the reaction, and to show the relative performance of different cases by plotting them together. This reaction plot is actually the integrated form of the kinetics (rate law), and shows how these change with time and reaction progress (substrate conversion). As explained further in the methodology section, the characteristic response of a reaction system to changes in reaction environment makes it possible to distinguish the dominating underlying phenomena in a given case.

One of the few, relatively well-established, graphical methods for reaction course analysis in biocatalysis was introduced by Selwyn and co-workers. ${ }^{30}$ The basis for their approach was to compare reaction trajectories, obtained using different biocatalyst concentrations, to identify enzyme inactivation over the course of the reaction. The rate of a reaction controlled purely by enzyme kinetics is dependent only on reaction composition and the amount of available catalyst. In the absence of other effects, the rate of reaction should therefore be directly proportional to the biocatalyst load. Deviation from this behavior is an indication that the reaction is additionally influenced by time-dependent effects.

Selwyn's approach is one example of a qualitative analysis of reaction data, which provides specific information about a reaction system (whether it is limited by catalyst stability or not) more effectively than the traditional quantitative approach. In this case, the catalyst load was modified to adjust the reaction trajectory - the plot of substrate conversion as a function of time. In Selwyn's approach the reaction trajectory is modified by adjusting the reaction time required to reach a given conversion. This allows the identification of time-dependent effects in the system with (ideally) only two experiments. A third outcome of this experiment is also possible: where the overall relative performance becomes worse at higher catalyst load. This can occur 
when the availability (solubility), or rather rate of supply, of substrate to the catalyst is limiting, and therefore would be an indication of mass transfer limitations in the reaction system.

The second parameter which has a predictable impact dependent on the dominating phenomena is the initial substrate load. As for the catalyst load, changing the substrate load will also generally change the time required to achieve a given conversion. This can be used to distinguish underlying mechanisms for the main limitations, and is explained further in the methodology section and demonstrated in the case studies.

In this study, only the impacts of catalyst and substrate load on the reaction trajectory have been considered. While other parameters such as $\mathrm{pH}$ and temperature could also be of interest, it is important to note that the method used here relies on a well-understood link between the varied parameter and the two fundamental variables used in the analysis: reaction time and conversion.

A final point regarding the use of reaction trajectories to distinguish between limiting phenomena is the normalization of plot axes to aid visual analysis. We propose that axes are normalized based on the parameter modified between experiments, such that for 'pure' behavior the plots superimpose on each other. The simplest example of this is the already mentioned Selwyn plot. Here, the time axis is normalized by multiplying it by the catalyst load, with the expected result that experimental series at different biocatalyst loadings will overlap if there are no time-dependent effects.

In order to investigate the effect of substrate concentration, it is also beneficial to normalize the axes based on substrate concentration. Reaction progress should thus be presented using a relative measure such as fractional conversion $(X)$, or reaction yield relative to a theoretical maximum. However, it is also necessary to normalize the reaction time (duration). This follows 
from the condition to normalize on 'pure' behavior: if the activity of the catalyst is independent of the substrate concentration, the time required to reach a given (normalized) reaction progress should be proportional to the amount of substrate to be converted.

SUGGESTED ORDER OF INVESTIGATION. As discussed, the primary limitations of a biocatalytic reaction system can be distinguished by analysis of reaction trajectories obtained through the systematic variation of key reaction parameters. First, limitations of enzyme kinetics, stability and mass transfer can be distinguished through the variation of catalyst load. Secondly, distinctions between underlying mechanisms can then be made by varying the substrate load. It is important to recognize that the underlying mechanism can be masked by the dominating limitation. For example, little or no useful information regarding the catalyst kinetics or stability can be gleaned from a substrate load experiment in a system that is primarily limited by mass transfer. We therefore suggest that the main limitation is first determined using the catalyst load experiment. If it turns out that mass transfer is the main limitation, the reaction system should be modified to address this limitation, before further investigation of the catalyst properties are attempted (e.g. changing the mixing conditions, scaling up the reaction (for improving gas-liquid mass transfer), ${ }^{33}$ or by implementing a reactor design that improves mass transfer ${ }^{34}$ ). Likewise, significant activity loss over the course of the reaction affects the analysis of kinetics. In order to ensure no secondary effects, the authors suggest that preliminary studies are carried out to ensure that the enzyme is stable over the course of the reaction trajectory measurements.

The impact of the two parameters (biocatalyst load and substrate concentration) on the different limitations is illustrated in Figure 1. For the purposes of the illustration, it has been assumed the catalyst kinetics are dominated by substrate binding to a certain point (up to the order of magnitude of $K_{M}$ ), beyond which it operates under fully saturated conditions until, above 
a certain concentration, the substrate or product causes inhibition. These two effects are independent of the enzyme concentration and therefore the limiting values run parallel to the yaxis. The catalytic rate is proportional to the enzyme load up to a point, beyond which substrate mass transfer can become a limiting factor. An increase in substrate load results in increased mass transfer, and consequently moves the limitation to catalyst load. Finally, the reaction can also be limited by catalyst stability. Since the reaction time will scale with the catalyst load (linearly under zero-order kinetics), the amount of enzyme required (in the absence of stability limitations) is proportional to the substrate load.

In the given example, the system is limited by different factors under different conditions, i.e. combinations of substrate and catalyst load. In this case, there is also a large domain where the rate of reaction is only limited by the $k_{\text {cat }}$ and catalyst load (commonly referred to as $V_{\max }$ $\left(=k_{c a t} \times\right.$ enzyme concentration $\left.)\right)$. Of course, not all of the indicated domains/limitations will necessarily be relevant in a given case. In an attempt to generalize, Figure 1 provides a conceptual blueprint of an expected regime map that can guide the changes in enzyme or substrate loading which an experimentalist should make in order to be able to evaluate the different bottlenecks. 


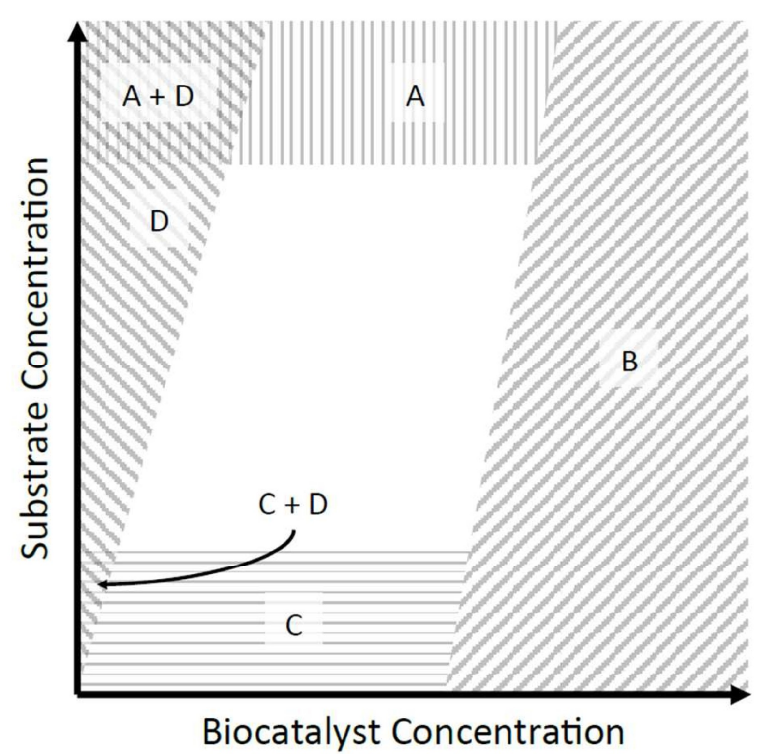

Figure 1. Conceptual regime map illustrating the different types of process limitations and how they relate to substrate and biocatalyst concentration (load). A: Inhibition, B: Mass transfer, C: Kinetically limited, D: Stability.

ENZYME KINETICS. Limitations in catalyst kinetics can be introduced through a number of mechanisms. In this study, these have been grouped as being related to substrate binding, competitive inhibition of substrate binding by the product, competitive inhibition by a second substrate, and non-competitive inhibition. The effect of these on the reaction trajectory is illustrated in the Methodology section.

To understand the impact of each kinetic mechanism, it is instructive to consider MichaelisMenten (single-substrate enzyme) kinetics, as shown in eq. 1:

$$
\frac{d S}{d t}=-\frac{k_{c a t} \cdot E(t) \cdot S}{K_{M}+S}=-\frac{k_{c a t} \cdot E(t)}{\frac{K_{M}}{S}+1} \quad \text { eq. } 1
$$

This is the standard Michaelis-Menten rate law, arranged to illustrate the interaction between the binding coefficient $\left(K_{M}\right)$ and the substrate concentration. The expression indicates that the 
rate of substrate consumption is directly proportional to the maximum activity of the catalyst $\left(k_{c a t}\right)$ and the concentration of active catalyst present $(E(t))$. In cases where $S>>K_{M}$, then $K_{M}$ can be neglected, making the rate effectively independent of the substrate concentration (zero order kinetics). On the other hand, when $S<<K_{M}$ the rate law tends towards first order kinetics (with rate constant $\left.k_{\text {cat }} / K_{M} \cdot E(t)\right)$.

A reaction limited by substrate binding is one where the substrate binding reduces the rate of reaction considerably over the course of the reaction. A similar effect can be observed for competitive inhibition by (co)substrate or product, which effectively acts to increase the (apparent) $K_{M}$. By contrast, non-competitive inhibition by either component will reduce the maximum activity of the catalyst, effectively reducing the (apparent) $k_{c a t}{ }^{26}$ The formal equations describing these effects for slightly more complex (substituted enzyme) kinetics are given in the Materials and Methods section.

ENZYME STABILITY. Loss of enzyme activity over the course of a reaction can be induced through a number of mechanisms, which potentially can be very complex. For the purposes of this study, we have considered three categories of inactivation: exponential decay (first-order relative to the concentration of active catalyst) independent of the reaction, exponential decay induced by the reaction product and total turnover number (TTN) related inactivation. ${ }^{35}$

Exponential, conversion-independent, decay of enzyme activity is related to random modifications to the protein structure that render the enzyme inactive. ${ }^{36}$ This can be, for example, unfolding of the protein or modification of sensitive key amino acids. ${ }^{37}$ These mechanisms can be strongly dependent on reaction parameters such as temperature, $\mathrm{pH}$ and the presence of solvents in the reaction system. As already mentioned this type of inactivation is first-order 
relative to the concentration of active enzyme, which gives the integrated exponential expression for remaining enzyme activity as shown in eq. 2 :

$$
E(t)=E_{0} \cdot e^{-k_{d} \cdot t} \text { eq. } 2
$$

where $t$ is the reaction time, $k_{d}$ is the rate constant for inactivation, $E_{0}$ is the initial enzyme concentration and $E(t)$ the enzyme concentration at time $t$.

Product-induced exponential decay is similar to the conversion-independent mechanism, however in this case the reaction product (or a linked component) acts to promote the inactivation in some way. In effect, $k_{d}$ in eq. 2 becomes dependent on the concentration of reaction product. In this study, a proportional relationship between stability and product concentration has been assumed, although other dependencies are possible.

The last mechanism (TTN related inactivation) does not imply that each catalyst molecule will convert a specific number of substrate molecules, but rather that the rate of decay is linked to the rate of catalysis. This is relevant for reactions whose catalytic mechanism produces intermediate species or catalyst conformations that are prone to result in catalyst inactivation. ${ }^{37}$

MASS TRANSFER LIMITATIONS. The last main type of limitation considered in this study is mass transfer, or rather the rate of substrate supply to the catalyst. It should be mentioned that for homogeneous batch reactions in a single (aqueous) phase with soluble enzyme, where both the catalyst and substrate(s) are homogeneously distributed throughout the reaction volume, mass transfer will not be limiting. However, mass transfer can quickly become limiting if the substrate is dosed, the reaction is poorly mixed (especially important at larger scale), product formation influences the viscosity of the reaction medium, the substrate is poorly water-soluble or if the substrate needs to move from an auxiliary phase (gas or liquid) to one where the reaction takes place. Mass transfer limitations are aggravated further if the enzyme is used in a heterogeneous 
formulation, for example in whole-cells or in an immobilized enzyme preparation. Depending on the type of mass transfer limitation, an accurate description of such a system can be very complicated and is often highly empirical. Nevertheless, the common effect of all mass transfer limitations (in the context of enzymatic reactions) is to reduce the specific activity of the catalyst, when the catalyst load is increased relative to the mass transfer capacity. Thus, the enzyme load experiment suggested in this study will distinguish when catalyst performance is significantly limited by mass transfer of substrate through the reaction media (or from another phase) to the catalyst.

Interestingly, the catalyst load experiment will not identify mass transfer limitations caused by immobilization or by the cell membrane for whole-cell catalysis. This is because the potential for mass transfer (to the catalyst surface and into the particle/through the cell membrane) scales directly with the catalyst load. Identifying these types of limitation requires changing the potential for mass transfer relative to the available catalytic activity, for example by immobilizing on smaller particles or opening the cells, which is outside of the scope of this study. For these biocatalysts it is the formulation as a whole, rather than the enzyme at its core, that is evaluated.

Since mass transfer limitations linked to the formulation of the catalyst cannot be identified in a catalyst load experiment, it will influence the characteristic behavior of the enzyme with respect both to its kinetics and stability. With respect to the kinetics, the effect is typically to increase the apparent $K_{M}$, meaning that non-zero order kinetics are typically more pronounced with immobilized (or whole-cell) formulations than with the free enzyme. This is because internal and/or external mass transfer limitations in such formulations will result in an average 
substrate concentration for the enzyme that is lower than in the bulk. As a result, a higher substrate concentration is required to fully saturate the catalyst. ${ }^{38,39}$

A reduced activity on immobilization relative to the soluble form of the enzyme can be expressed in terms of an effectiveness factor, which is a function of the intrinsic enzyme kinetics relative to the potential for mass transfer for the preparation. ${ }^{40}$ While a low effectiveness factor means that the full potential of the enzyme cannot be realized in terms of reaction rate, it can also lead to an increased (apparent) stability relative to soluble enzyme. This is a result of the effectiveness factor increasing as the amount of active enzyme is reduced over time relative to the potential for mass transfer. ${ }^{41}$

\section{Methodology}




\section{1}

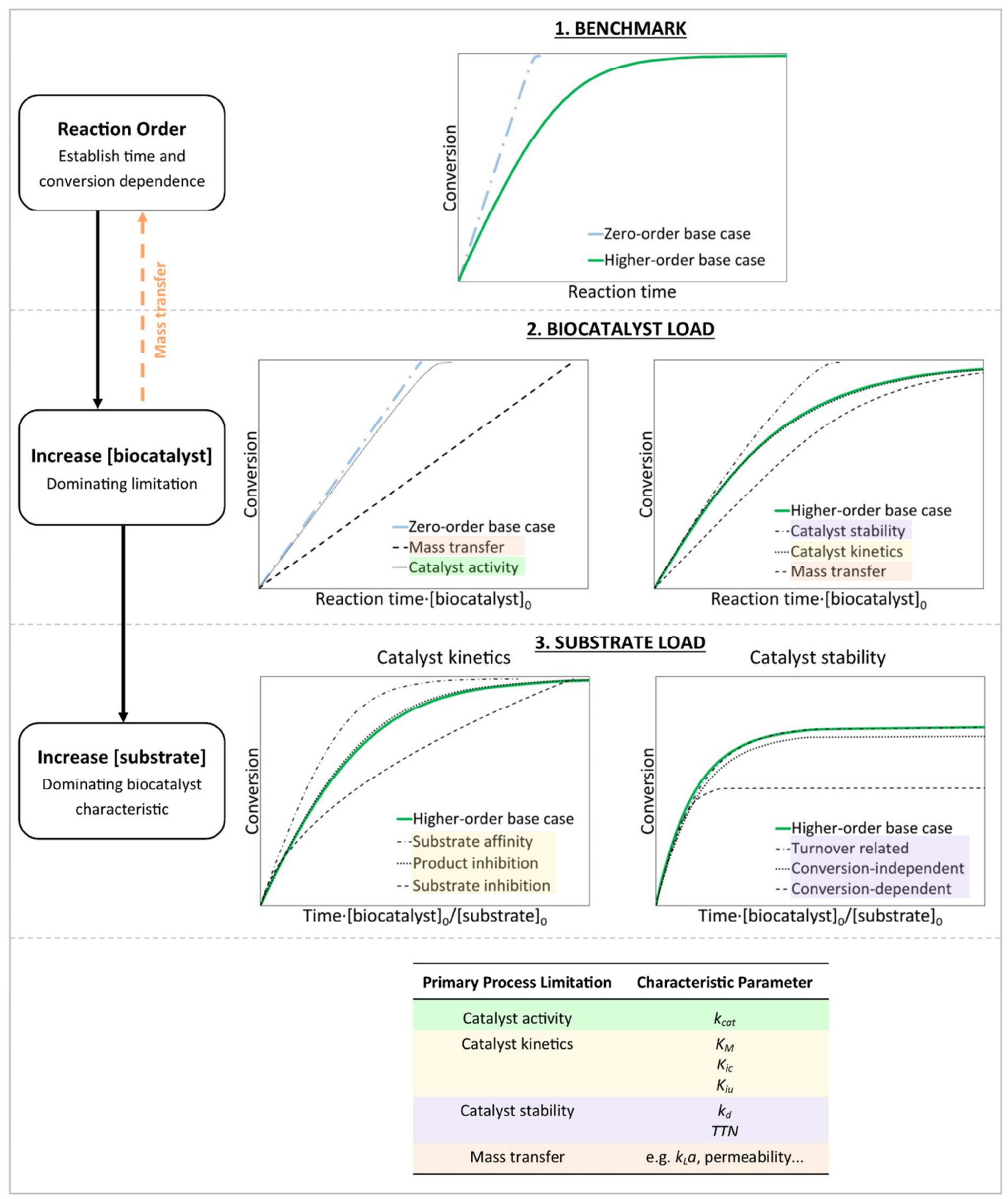

Figure 2. Illustration of the methodology to establish process and catalyst limitations. 1 . Benchmark experiment to determine reaction order under given conditions; 2. Biocatalyst load experiment to determine process limitations; 3 . Substrate load experiment to determine catalyst 
limitations. Trajectories show an increase in the varied parameter. Catalyst activity limited: $k_{c a t}$ turnover number; Kinetically limited: $K_{M}$ - Michaelis constant, $K_{i c}$ - competitive inhibition constant, $K_{i u}$ - uncompetitive inhibition constant; Enzyme stability limited: $k_{d}$ - first-order rate constant for inactivation, TTN - total turnover number; Mass transfer limited: $k_{L} a$ - overall volumetric mass transfer coefficient.

RELEVANT CONDITIONS. Figure 2 shows the developed methodology for identifying reaction bottlenecks. The reaction plots have been generated using models based on modifications of GOx reaction kinetics (see Materials and Methods). The methodology assumes that a relevant starting point, in terms of reaction conditions, has already been identified. However, what is relevant depends very much on the case and the objective of reaction development. The guidelines themselves for achieving a given reaction performance are outside the scope of this study. Nevertheless, the choice of reaction conditions will dictate the main observable phenomena (e.g. effects of inhibition will only be seen at high substrate loading). We propose that, as far as possible, reaction conditions should reflect those of the envisaged industrial process. For example, if high substrate concentrations and the addition of co-solvents are required for downstream separations, then these should also be incorporated at this early stage of reaction characterization.

THREE EXPERIMENTS. The methodology relies on three key experiments, all of which follow the conversion (reaction trajectory) over time. The first of these is to be used as a benchmark and is the reaction data collected for a single experiment using the conditions established as relevant for the case. This experiment establishes the general type of reaction behavior; specifically, if the rate of reaction varies with time and/or conversion or not (zeroorder kinetics). In the second experiment, the catalyst load is varied relative to the first 
experiment, and the impact is assessed in order to identify the main limitation in the system (in all of the example plots in Figure 2, the variable of interest is increased relative to the benchmark). Specifically, this experiment distinguishes between limitations related to the catalytic activity (through kinetics or stability) and limitations not related to the catalyst, such as mass transfer limitations. In the third type of experiment, the substrate concentration is varied again, relative to the baseline experiment. This experiment applies to systems limited by either enzyme kinetics or stability, and gives an indication of the underlying mechanism in either category.

1. BENCHMARK EXPERIMENT (ZERO- VS. HIGHER-ORDER KINETICS). The methodology starts with a single reaction trajectory experiment, meant to assess the general behavior of the reaction at the chosen conditions. In this analysis, reactions exhibiting a constant reaction rate throughout the course of reaction are classed as having zero-order kinetics. Under the conditions of the experiment, this type of behavior implies that no change in substrate/product composition (through enzyme kinetics) occurs, nor changes in reaction time (through inactivation). Conversely, a reaction that exhibits a reaction rate that changes over the course of the reaction is classified as having higher-order kinetics. This behavior also includes all reactions that fail to reach completion (relative to the reaction equilibrium). It is worth noting that it is common for reactions to show zero-order behavior initially and transition into higherorder kinetics at higher conversion.

\section{CATALYST LOAD EXPERIMENT (IMPACT OF CATALYST LOAD - TIME} DEPENDENCE). For the second step of the methodology, the catalyst load is varied in order to separate the three main classes of limitation (catalyst kinetics, catalyst stability or non-enzyme related). As explained in the theory section, in a reaction controlled entirely by enzyme kinetics 
the reaction rate is independent of the reaction time. Because of this, plots of reaction courses with different catalyst loads should superimpose when the reaction time is normalized by the catalyst load.

In a system limited by catalyst stability, the reaction rate should vary less with conversion at higher catalyst loads. This is because inactivation is a time-dependent process, and therefore less inactivation will have occurred in a reaction that proceeds more quickly.

Finally, in some cases the specific reaction rate will be lower at higher catalyst loadings. This is because something other than intrinsic catalyst kinetics (or stability) limits the reaction, typically the rate of substrate mass transfer between phases or compartments in a reaction system. Indeed any further analysis of the catalyst performance will be hindered by extensive mass transfer (non-catalyst related) limitations. If such an analysis is desired, reaction conditions should be modified to either improve mass transfer, or to reduce the need for high mass transfer by reducing the catalyst load (as indicated in Figure 2). Assessment should then be done from the beginning with a new baseline experiment again.

A reaction exhibiting zero-order behavior is not significantly affected by catalyst inactivation during the course of the reaction and is saturated by the substrate throughout the reaction. Furthermore, improving such a reaction necessitates improving the maximum activity of the catalyst. The specific improvement will depend on the reaction system and catalyst formulation, and is discussed further in the Discussion section.

\section{SUBSTRATE LOAD EXPERIMENT (IMPACT OF SUBSTRATE LOAD - CONVERSION DEPENDENCE). In the third step of the methodology, the substrate concentration is varied to distinguish between mechanisms for both kinetic and stability limitations. Changing the substrate concentration directly modifies the conditions for the}


catalyst: the starting point for the reaction and consequently the entire reaction course will be different. Additionally, changing the substrate concentration also indirectly changes the duration of the reaction: more substrate typically takes longer to convert with a given amount of catalyst.

In those cases the reaction is primarily limited by catalyst kinetics, it can then be of interest to determine the dominant kinetic limitation. For soluble enzyme catalysis in a homogeneous system exhibiting higher-order behavior, the rate of reaction will depend solely on the enzyme kinetics. These will be linked to either a high saturation constant for the substrate (i.e. high $K_{M}$ ), or competitive or non-competitive inhibition by one or more of the reaction components. To distinguish between these effects, reaction data for different substrate loads should be compared in a plot of conversion against reaction time normalized by both enzyme and substrate load.

In this example, for a reaction that becomes slower at higher conversions due to a high $K_{M}$ for the substrate, the rate of conversion will become disproportionately faster at higher substrate concentrations. This is because the catalyst becomes more saturated at a given conversion with more substrate. On the other hand, if the kinetic limitation is due to competitive inhibition by the product, the combined effect of inhibition and increased substrate load results in an overlap of the reaction data in the normalized plot. Finally, non-competitive inhibition by the product results in a disproportionately lower rate of conversion at increased substrate concentrations (this effect can also be seen when one substrate acts as a competitive inhibitor for another in a twosubstrate reaction)

If it turns out that the reaction rate diminishes over the course of a reaction due to inactivation of the enzyme, it could be of interest to determine how this activity is lost. This study considers three mechanisms for inactivation: (1) conversion-independent stability where the rate of inactivation is proportional to the concentration of remaining active enzyme (and linked to a 
parameter that is fixed in the experiment); (2) conversion-dependent stability where the rate of inactivation varies with the concentration of enzyme and the concentration of a reaction component (for example product-induced inactivation); and (3) Total turnover number (TTN) type-stability where the rate of inactivation is proportional to the rate of reaction.

For soluble enzyme catalysis in a homogeneous system, the performance of a reaction limited by product (or substrate) induced inactivation will become progressively worse at higher substrate loads in a plot of conversion vs time normalized by catalyst and substrate load. If the inactivation is instead linked to TTN or conversion-independent inactivation, the reaction curves for different substrate loads will superimpose in the normalized plots. These two inactivation mechanisms cannot be separated in this type of experiment if stability is the only limiting factor.

\section{Results and Discussion}

In the following section, the methodology has been applied to three separate case studies. Each individually shows how the method can distinguish different process performance limitations.

GLUCOSE OXIDASE (GOx). GOx catalyzes the oxidation of glucose to glucono-lactone, which (at neutral $\mathrm{pH}$ ) spontaneously forms gluconic acid, and the by-product hydrogen peroxide (Scheme 1). Oxygen is required to perform the reaction and half the stoichiometric requirement is supplied in the form of molecular oxygen by bubbling air into the bioreactor, the residual by decomposing hydrogen peroxide using added catalase.

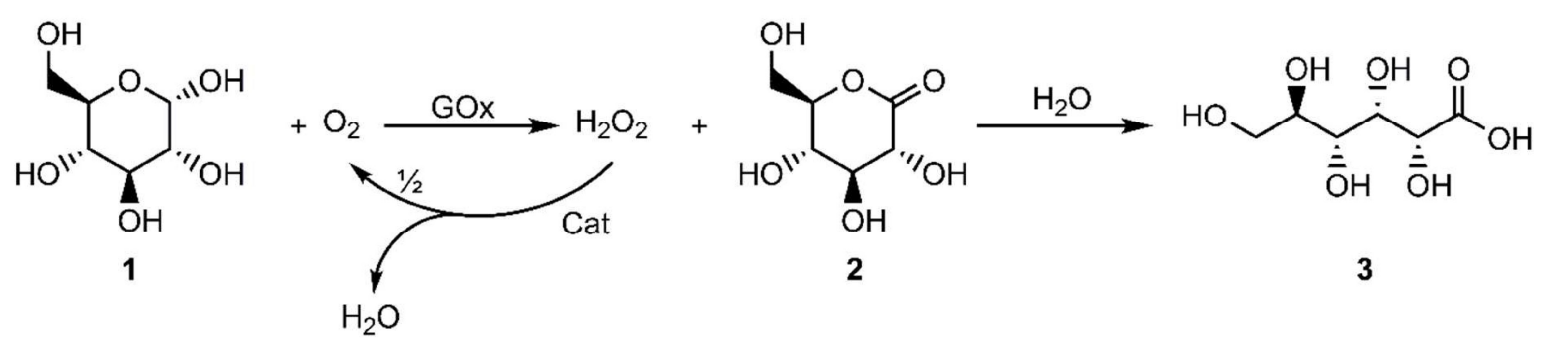


Scheme 1. Oxidation of glucose (1) to glucono-lactone (2), which spontaneously hydrolyses to gluconic acid (3) at $\mathrm{pH} 7.5$, and includes the generation of hydrogen peroxide. The reaction is catalyzed by glucose oxidase (GOx) and the hydrogen peroxide is decomposed to water and oxygen by catalase (Cat).

An initial experiment with low enzyme and substrate concentration indicated first-order reaction behavior (base case, Figure 3). A second experiment, doubling the biocatalyst load and keeping the same glucose concentration, showed no sign of enzyme inactivation and revealed that the reaction was kinetically limited (Figure 4). To identify the dominating kinetic limitation under these conditions, the glucose load was increased to $20 \mathrm{mM}$; the results revealed that the reaction was limited by substrate affinity ( $K_{M}$ limitation, Figure 5$)$. It turns out that the reaction was carried out at a substrate concentration beneath the Michaelis-Menten constant for glucose, showing a conversion/time-dependent reaction.

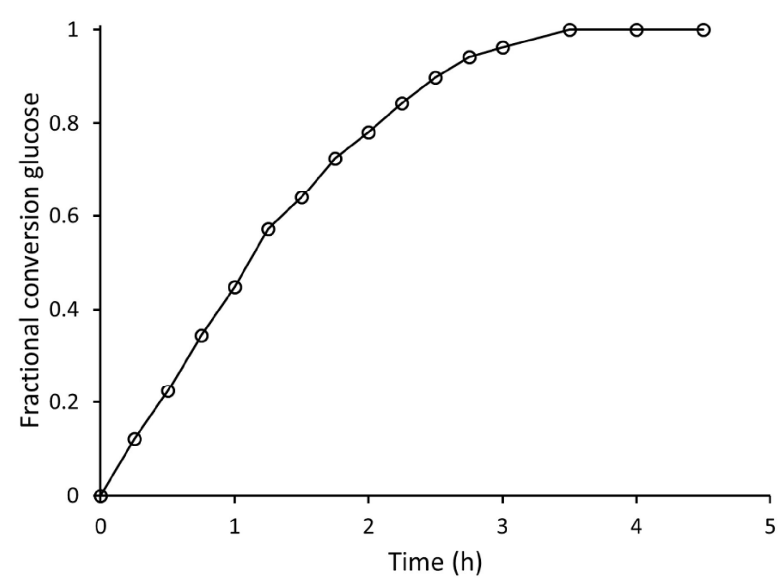

Figure 3. Biooxidation of $10 \mathrm{mM}$ of glucose to gluconic acid at $25^{\circ} \mathrm{C}$ and $\mathrm{pH} 7.5$ in an aerated stirred tank reactor (500 rpm and $1 \mathrm{vvm}$ of air) with $10 \mathrm{mg} / \mathrm{L}$ of GOx. 


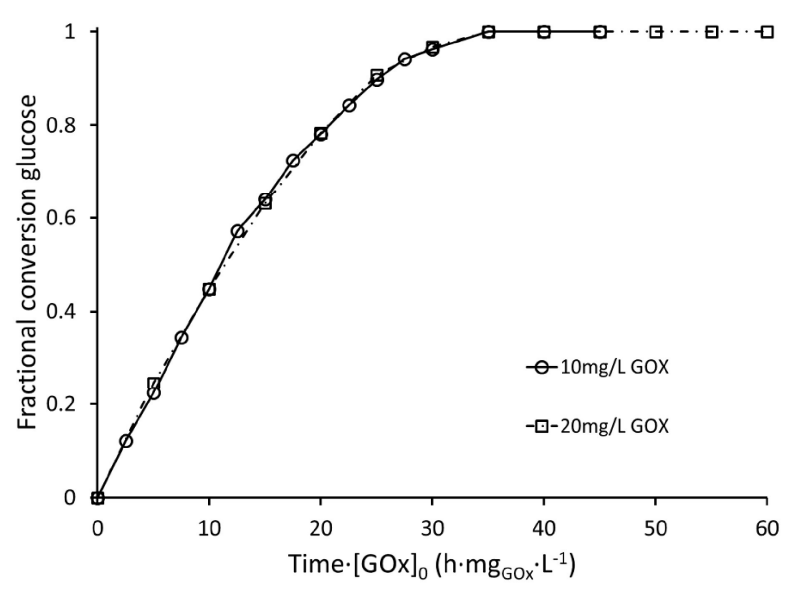

Figure 4. Effect of biocatalyst load on the biooxidation of $10 \mathrm{mM}$ of glucose with $10(\mathrm{O})$ and $20(\square) \mathrm{mg} / \mathrm{L}$ of GOx.

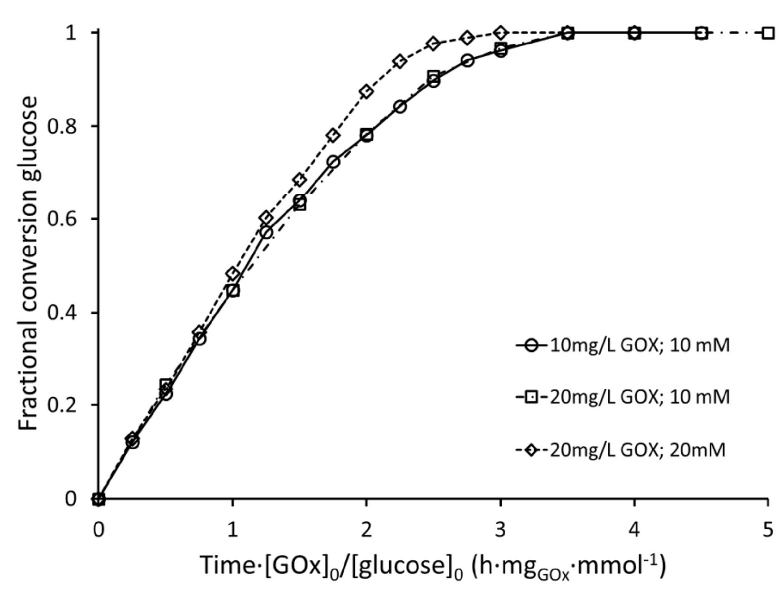

Figure 5. Effect of increased substrate load on the biooxidation of glucose $(10 \mathrm{mM})$ at varying concentrations of enzyme (10 mg/L of GOx (०), $20 \mathrm{mg} / \mathrm{L}$ of GOx (口)) and $20 \mathrm{mM} \mathrm{(20} \mathrm{mg/L} \mathrm{of}$ $\operatorname{GOx}(\diamond))$.

To eliminate kinetic limitations related to $K_{M}$, an experiment with higher glucose concentration (200 mM) was conducted and these results exhibited zero-order reaction behavior (Figure 6). Following the methodology, the biocatalyst load was increased and an improvement in reaction rate was observed (Figure 7). However, the rate enhancement going from 50 to $100 \mathrm{mg} / \mathrm{L}$ of 
biocatalyst was not proportional, thus indicating mass transfer limitations under these conditions. For this specific reaction, the mass transfer limitation is related to the oxygen supply into the reactor. Under these aeration and agitation rate conditions it is not possible to increase the oxygen transfer rate from gas to liquid phase further. Achieving substantially higher reaction rates would require an increase in scale (since gas-liquid mass transfer typically improves with scale), ${ }^{33}$ an improvement in the aeration/agitation system or an increase in the (partial) pressure of oxygen used for aeration, which would increase the driving force for oxygen mass transfer.

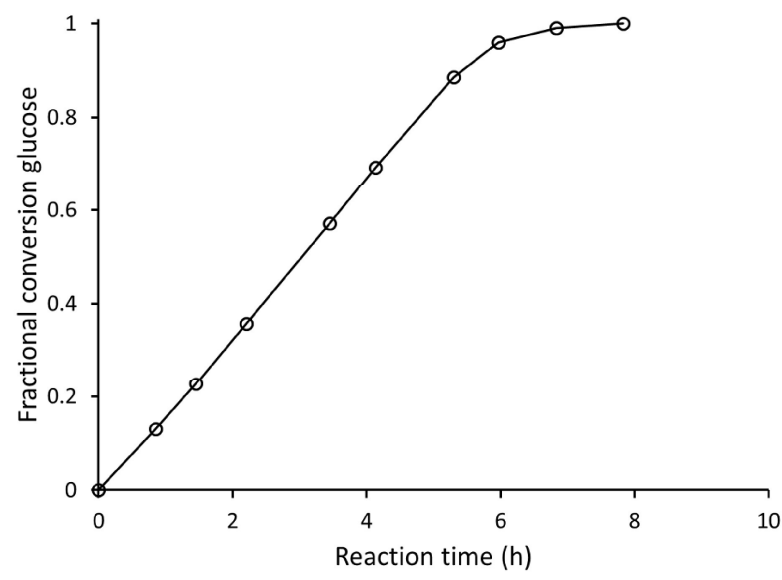

Figure 6. Biooxidation of $200 \mathrm{mM}$ of glucose to gluconic acid at $25^{\circ} \mathrm{C}$ and $\mathrm{pH} 7.5$ in an aerated stirred tank reactor (500 rpm and 1 vvm of air) with $50 \mathrm{mg} / \mathrm{L}$ of GOx.

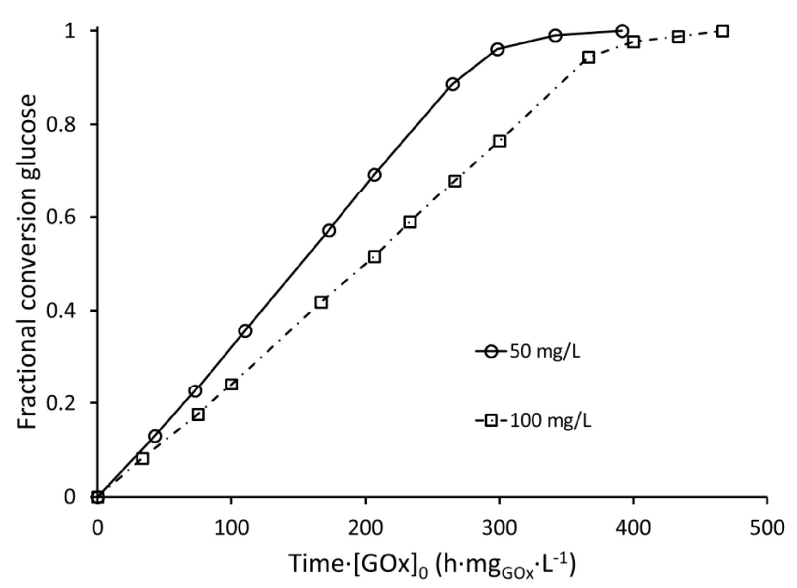


Figure 7. Effect of biocatalyst load on $200 \mathrm{mM}$ of glucose conversion with 50 (०) and $100(\square) \mathrm{mg} / \mathrm{L}$ of GOx.

MONOAMINE OXIDASE (MAO). The second case study is based on the selective oxidation of a bicyclic amine to the corresponding imine (Scheme 2), catalyzed by monoamine oxidase (MAO) from Aspergillus niger. A separate characterization of this reaction has been published previously. ${ }^{42}$ The intended application of the studied reaction is in the synthesis of the target product as an API, and the reaction is of general interest for the chiral resolution of secondary amines.
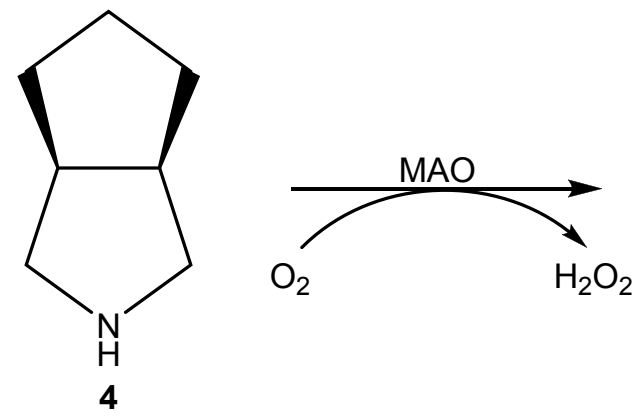
dominating underlying phenomena, the catalyst load was varied (Figure 9). Interestingly, the experiments where high catalyst loads were applied exhibited specific rates that were independent of the catalyst load (hence, a reaction primarily limited by catalyst kinetics). However, the experiment with lowest catalyst load $\left(12.5 \mathrm{~g}_{\mathrm{cww}} / \mathrm{L}\right)$ gave poorer performance at high conversion, indicating catalyst inactivation. To investigate the two effects further, two sets of substrate load experiments were carried out at high $\left(200 \mathrm{~g}_{\mathrm{cww}} / \mathrm{L}\right)$ and low $\left(12.5 \mathrm{~g}_{\mathrm{cww}} / \mathrm{L}\right)$ catalyst loads. The substrate load experiments with low catalyst load are shown in Figure 10. The reactions illustrate an increasingly pronounced trend to slow down with conversion at higher substrate concentration, indicating a conversion (or product) dependent inactivation mechanism. This is consistent with the previously published study.

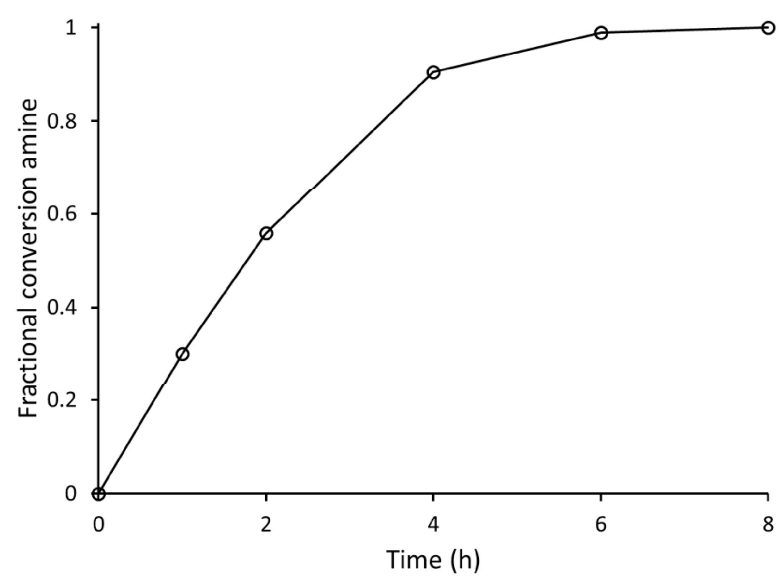

Figure 8. Selective oxidation of $50 \mathrm{mM}$ aza-biocyclo-octane by $200 \mathrm{~g}_{\mathrm{cww} / \mathrm{L}} \mathrm{MAO}$ at $37^{\circ} \mathrm{C}$. $500 \mathrm{mM}$ phosphate buffer was used to maintain $\mathrm{pH}$ 7.6. 


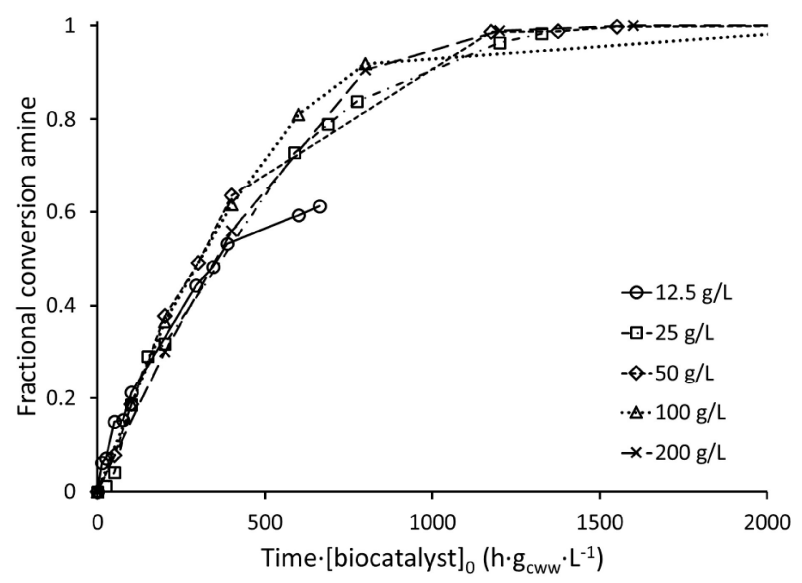

Figure 9. Effect of biocatalyst concentration in the selective oxidation of aza-bicyclo-octane. Catalyst loads of $12.5(\circ), 25(\square), 50(\diamond), 100(\triangle)$ and $200(\times) \mathrm{g}_{\mathrm{cww}} / \mathrm{L}$ whole-cells were used, other conditions as in Figure 8.

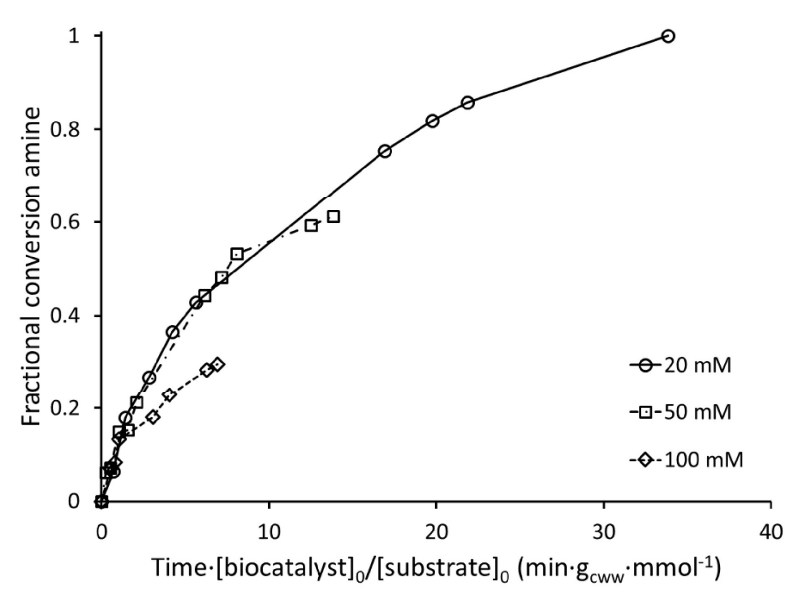

Figure 10. Effect of substrate concentration in the selective oxidation of aza-bicyclo-octane at low catalyst load $\left(12.5 \mathrm{~g}_{\mathrm{cww}} / \mathrm{L}\right)$. Substrate concentrations of $20(\odot), 50(\square)$ and $100(\diamond) \mathrm{mM}$ were used, other conditions as in Figure 8.

The substrate load experiments at high catalyst load are shown in Figure 11. The results illustrate a trend of improved relative rate of conversion with increased substrate loads, which indicates enzyme kinetics limited by a high apparent substrate binding coefficient $\left(K_{M}\right)$. 


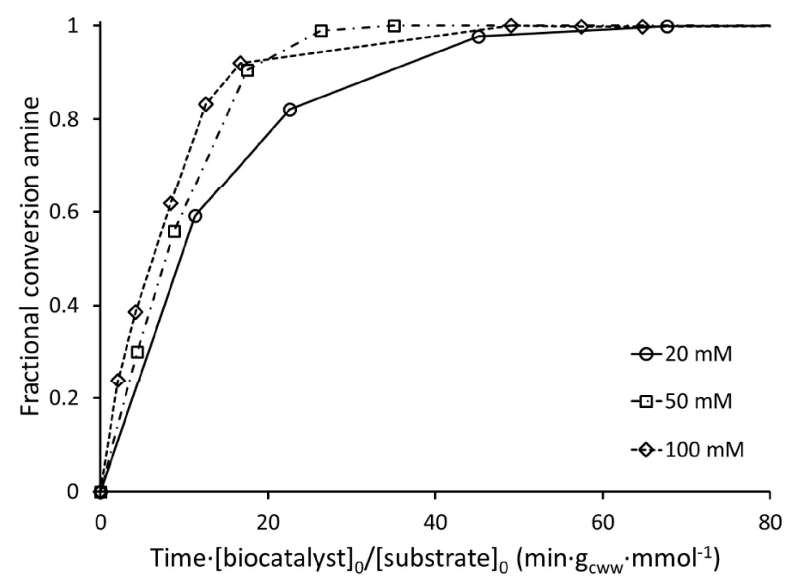

Figure 11. Effect of substrate concentration in the selective oxidation of aza-bicylo-octane at high catalyst load $\left(200 \mathrm{~g}_{\mathrm{cww}} / \mathrm{L}\right)$. Substrate concentrations of $20(\odot), 50(\square)$ and $100(\diamond) \mathrm{mM}$ were used, other conditions as in Figure 8.

BIODIESEL PRETREATMENT BY IMMOBILIZED LIPASE. The final case study is based on the esterification of oleic acid with methanol using an immobilized lipase (Scheme 3). The reaction has been reported previously. ${ }^{43}$ The intended application for the underlying study was the selective esterification of free fatty acids (FFAs) in vegetable oil as a pretreatment step for alkali-catalyzed biodiesel production (which requires a low FFA content to avoid excessive soap formation). While the glycerides in the vegetable oil feedstock are also substrates for the lipase, the esterification of FFA proceeds at a rate of 20 to 100 -fold that of the competing transesterification reaction. 


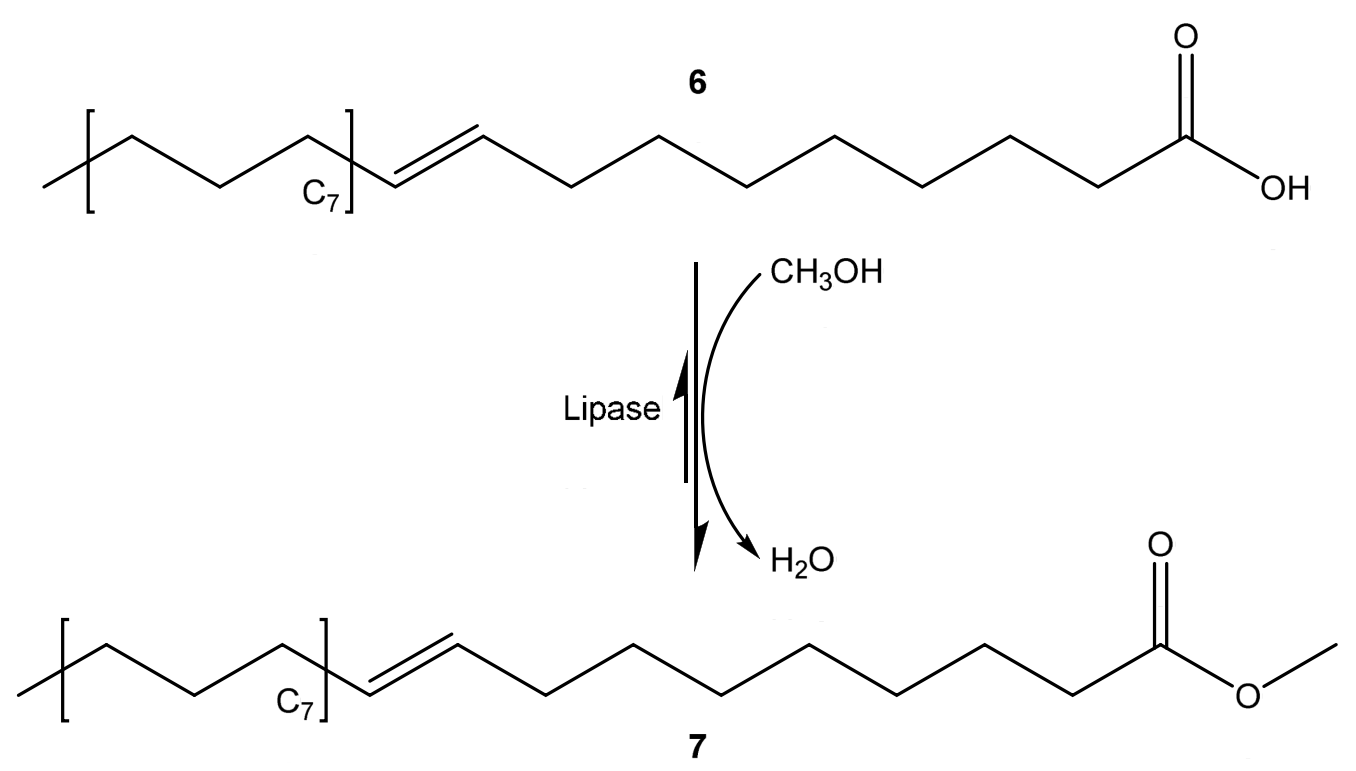

Scheme 3. Esterification of oleic acid (6) to methyl oleate (7) with methanol. The reaction is catalyzed by immobilized Candida antartica lipase B (N435).

It was determined that a methanol concentration of approximately $4 \%(\mathrm{v} / \mathrm{v})$ was required to achieve a sufficiently low FFA content with an initial FFA concentration of 5\% (which was deemed to be representative of low-quality oils of interest), using 5\% (w/v) catalyst to complete the reaction within an hour. In the following example, the methodology presented in this study has been applied to identify the primary bottleneck for a pretreatment process operated with these suggested parameters.

The standard reaction course indicates a conversion and/or time-dependent reaction rate (Figure 12). The catalyst load was varied to establish the main type of limitation in the system (Figure 13). The analysis indicates that the specific reaction rate is independent of the catalyst load within the studied range, which means that the reaction trajectory is primarily dictated by the kinetics of the catalyst (and thus purely conversion-dependent). Finally, the substrate load was varied to identify the dominating kinetic limitation in the system. Because the initial 
concentration of both oleic acid and methanol can be easily controlled in this system, two sets of experiments were conducted where each substrate was varied. Note that in both experiments, conversion is based on the oleic acid concentration as this is the limiting substrate. Likewise, the reaction time is normalized using the oleic acid concentration, to match the conversion.

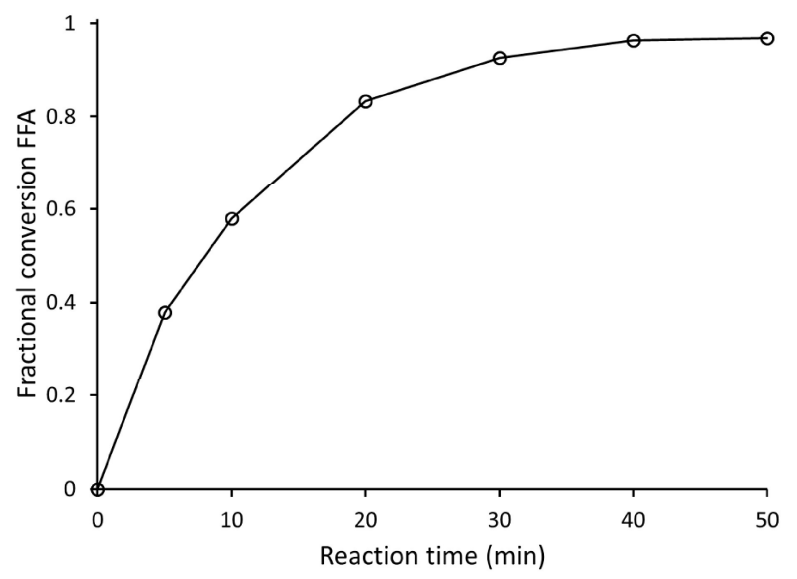

Figure 12. Selective esterification of $5 \%(\mathrm{v} / \mathrm{v})$ oleic acid and $4 \%(\mathrm{v} / \mathrm{v})$ methanol in vegetable oil at $45{ }^{\circ} \mathrm{C}$ using $5 \%(\mathrm{w} / \mathrm{v})$ immobilized CalB (N435).

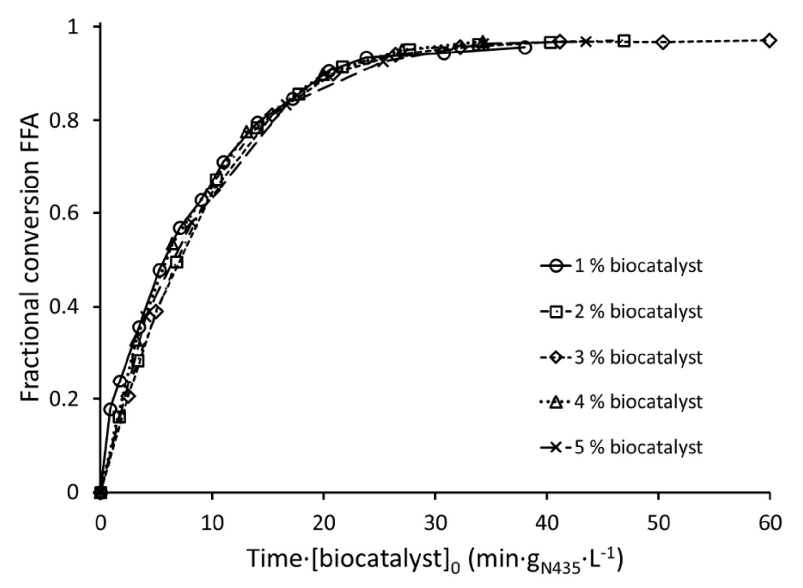

Figure 13. Effect of biocatalyst concentration in the selective esterification of oleic acid and methanol in vegetable oil. Catalyst loads of $1 \%(\circ), 2 \%(\square), 3 \%(\diamond), 4 \%(\triangle)$ and $5 \%(\times)(\mathrm{w} / \mathrm{v})$ N435 were used, other conditions as in Figure 12. 
As illustrated in Figure 14, methanol had a slight negative impact on the reaction trajectory. This indicates an inhibitory effect by this substrate, possibly through interfering in the binding of oleic acid. The series of experiments with increasing initial oleic acid concentrations exhibit a trend of increasing specific activity throughout the reaction, indicating that the catalyst is limited by a high (apparent) $K_{M}$ for this substrate (Figure 15). This also lends support to the hypothesis that methanol acts as a competitive inhibitor for oleic acid. Further experiments in the original study revealed that this was indeed the case; the performance of the reaction benefited from higher concentrations of both substrates as long as they were balanced against one another. ${ }^{43}$

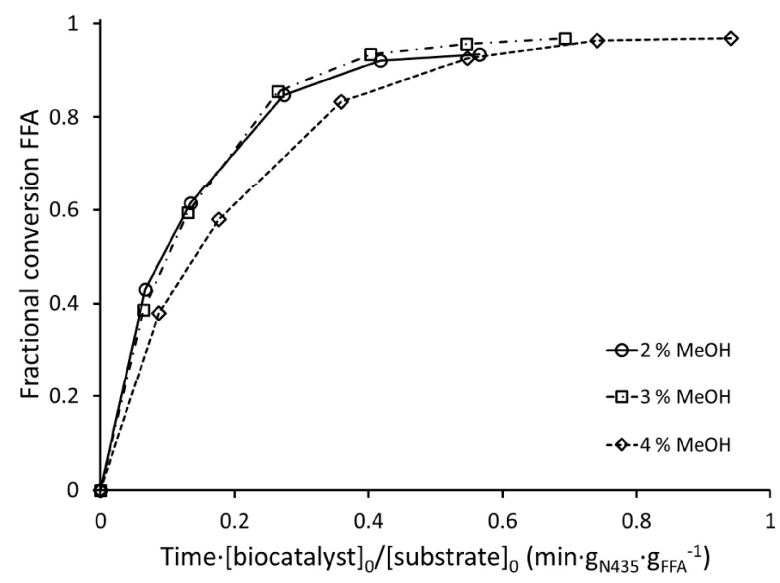

Figure 14. Effect of methanol concentration in selective esterification of oleic acid and methanol in vegetable oil. Methanol concentrations of $2 \%(\circ), 3 \%(\square)$ and $4 \%(\diamond)(v / v)$ were used, other conditions as in Figure 12. 


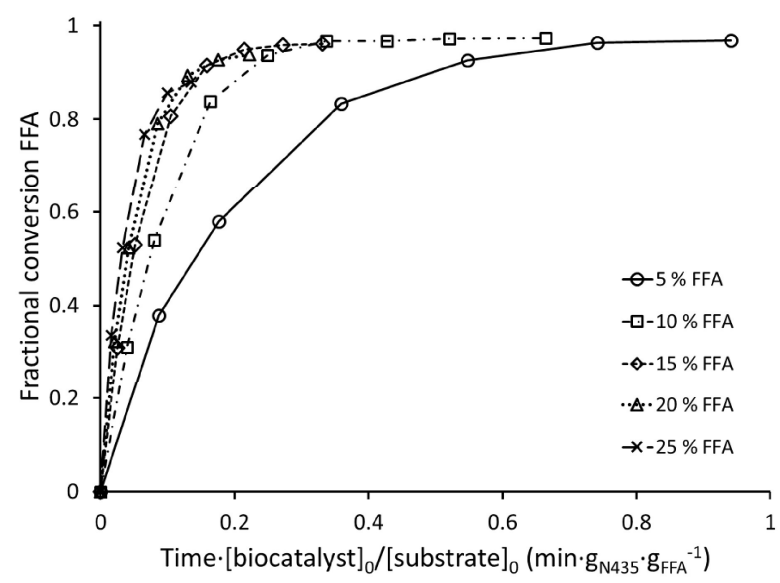

Figure 15. Effect of oleic acid concentration in selective esterification of oleic acid and methanol in vegetable oil. Oleic acid concentrations of $5 \%(\circ), 10 \%(\square), 15 \%(\diamond), 20 \%(\square)$ and $25 \%(\times)$ were used, other conditions as in Figure 12.

\section{Perspectives on the Methodology}

In the context of process development, once the limiting factor for process performance has been identified, the next logical step is to address that limitation. When the main limitation is enzyme kinetics or stability, there is potential for improvement by modifying enzyme through protein engineering. Such modifications can improve the affinity for the substrate (reducing $K_{M}$ ) or improve the stability of the protein. An alternative (or complimentary) approach in cases where the substrate or product has a negative impact on performance is to control the concentration of these reaction components, through controlled substrate supply (e.g. feeding) or in-situ product removal strategies, respectively. ${ }^{44}$ In cases where mass transfer is limiting, the most direct solution is to consider the reaction and reactor design: how substrate is introduced and transported to the enzyme, the choice of any co-solvents and choice of reactor configuration. Finally, in some cases (regardless of the limitation) the formulation of the catalyst should also be considered. The choice between applying whole-cell catalysts, crude (or purified) soluble- 
enzyme solutions or an immobilized preparation can have a great impact on both the activity and the stability of the catalyst. On the other hand, the choice of formulation also comes with a cost that can affect the feasibility of the process. ${ }^{45}$ Interestingly, this methodology might also be useful for benchmarking enzyme formulations with different specific activities in order to assess their suitability for process implementation.

It is important to understand that an indication of kinetic or stability limitations for a heterogeneous (e.g. whole-cell or immobilized) biocatalyst applies to the catalyst as a whole, rather than the specific enzyme. Catalyst kinetics and stability for such preparations are a combined effect of the underlying effects of enzyme kinetics and stability, and any mass transfer limitations that may apply to the catalyst itself (i.e. external and internal mass transfer (in)to a particle or permeability of a cell membrane). As a consequence, the kinetics and the stability of an enzyme can be quite different in the soluble form compared to the behavior exhibited by a whole-cell or immobilized preparation. It is not possible to discern these effects with the present methodology without modifying the preparation in such a way as to change the relevant mass transfer effects (e.g. by changing particle size or increasing permeability).

The simulated reactions used to illustrate the methodology (Figure 2) are based on reaction kinetics for an irreversible reaction that will go to completion, barring total loss of activity due to inactivation. However, the suggested methodology is fully applicable to thermodynamically limited reactions as well. The impact of catalyst load will be the same for an equilibrium reaction as for a non-reversible one. Further, varying the substrate load should not, for changes within an order of magnitude, drastically alter the equilibrium conversion in most cases. However, care should be taken when choosing substrate concentrations when studying two-substrate 
equilibrium reactions, because changing the concentration of only one of the substrates in such a case can change the expected conversion.

The methodology presented in this work is intended as a complement to established experiment-based evaluation methods that are used to support process development. Ultimately, the best tool for the task at hand should be used; a single end-point measurement can thus be sufficient to verify that the reaction achieves the required performance in some cases. On the other hand, if detailed information regarding the kinetic parameters of the catalyst is required (for example to evaluate the success of a specific modification to the protein), an initial rate experiment under carefully controlled conditions is likely the most pragmatic option. The methodology presented here is thus meant to provide an alternative, limited-effort approach in cases where more qualitative information is required; to provide a sense of the characteristics of the reaction system of interest under process conditions and to guide further experimentation and development work.

\section{Conclusions}

The methodology presented in this study provides a fast and efficient means of characterizing the performance and limitations of a biocatalytic reaction system under given conditions, with minimal experimental effort. This is useful for bottleneck identification in support of further development work, but we would argue that this type of initial characterization should also precede any in-depth scientific investigation. For example, it is important for any study of enzyme kinetics to ensure that the intrinsic kinetics dictate reaction performance, rather than catalyst stability or substrate mass transfer.

\section{Materials and Methods}


SIMULATIONS: REACTION KINETICS AND STABILITY MODELS. The simulated reaction trajectories used to illustrate the methodology presented in this study are based on substituted enzyme (ping-pong bi-bi) kinetics, ${ }^{26}$ using kinetic parameters determined for a glucose oxidase (GOx)-based reaction. The simulations also implement models for enzyme inactivation, with parameters set manually to produce characteristic behavior. Finally, a basic model for oxygen transfer from gas to liquid phase (based on $k_{L} a$ ) has been implemented using parameters determined in our laboratory, which correspond to what can be found in literature. ${ }^{46}$

GOx-catalyzed oxidation of glucose was simulated using the model shown in eq. 3 :

$$
\frac{d S}{d t}=-\frac{k_{c a t} \cdot E \cdot O_{2}}{\frac{K_{M S} \cdot O_{2}+K_{M O}+O_{2}}{S}} \quad \text { eq. } 3
$$

where $S, \mathrm{O}_{2}$ and $E$ are the molar concentrations of glucose, dissolved oxygen and active enzyme, respectively. $k_{c a t}$ is the apparent maximum specific activity of the enzyme, $K_{M O}$ is the binding constant for oxygen and $K_{M S}$ the apparent binding constant for glucose.

Competitive and non-competitive inhibition were simulated by introducing apparent constants for $K_{M S}$ and $k_{\text {cat }}$, shown in eq. 4 and 5:

$$
\begin{gathered}
k_{c a t, a p p}=\frac{k_{c a t}}{1+\frac{i}{K_{i u}}} \quad \text { eq. } 4 \\
K_{M S, a p p}=K_{M S} \cdot \frac{\left(1+\frac{i}{K_{i c}}\right)}{\left(1+\frac{i}{K_{i u}}\right)} \quad \text { eq. } 5
\end{gathered}
$$

where $i, K_{i c}$ and $K_{i u}$ are the inhibitor concentration (which can be equivalent to the substrate or product concentration) and competitive and uncompetitive inhibition constants, respectively. ${ }^{26}$

Gradual loss of activity was simulated by applying rate expressions for the decay of active enzyme. The simplest model applied was a first order (with respect to active enzyme concentration) rate model shown in eq. 6 : 


$$
\frac{d E}{d t}=-k_{d} \cdot E \quad \text { eq. } 6
$$

where $k_{d}$ is the rate constant for inactivation. This model results in classical exponential decay of the available active enzyme.

For a conversion-dependent inactivation rate (relevant for example when the reaction product has a deleterious effect on the enzyme), an expression that is first-order with respect to both active enzyme concentration and an inactivating agent $D$, shown in eq. 7:

$$
\frac{d E}{d t}=-\left(k_{d, X} \cdot D\right) \cdot E \quad \text { eq. } 7
$$

For product-dependent inactivation, $D$ would be equal to the product concentration.

Total turnover type inactivation was also considered. In this case, the rate of inactivation is linked to the rate of reaction, such that a certain fraction of catalytic cycles result in an inactivated enzyme. The characteristic measure for this type of inactivation is the total turnover number $(T T N)$, which describes the average number of catalytic cycles (turnovers) for every instance of inactivation, as shown in eq. 8:

$$
\frac{d E}{d t}=\frac{1}{T T N} \cdot \frac{d S}{d t} \quad \text { eq. } 8
$$

The final type of kinetics that was considered was the rate of supply of a substrate from an auxiliary phase (mass transfer limitation). The rate of mass transfer is typically proportional to the difference in chemical activity between the two volumes (potential) and the area available for the flux, and inversely proportional to the characteristic distance. The model chosen to exemplify this describes the rate of oxygen mass transfer (OTR) from gas to liquid phase in an aerated system as shown in eq. 9:

$$
O T R=k_{L} a \cdot\left(O_{2}^{*}-O_{2}\right) \quad \text { eq. } 9
$$

where $\mathrm{O}_{2}$ is the dissolved oxygen concentration, $\mathrm{O}_{2}{ }^{*}$ is the saturation concentration of oxygen in the liquid phase at given conditions (e.g. temperature, partial pressure of oxygen in the gas 
phase with which it is in equilibrium) and $k_{L} a$ the oxygen mass transfer coefficient, which is an empirically derived parameter for the experimental setup (linked to reactor dimensions, aeration rate and agitation power input. An additional rate expression was required to describe the change of oxygen in the system, shown in eq. 10:

$$
\frac{d O_{2}}{d t}=O T R+\frac{1}{2} \cdot \frac{d S}{d t} \text { eq. } 10
$$

which is the sum of oxygen supplied to the system and the oxygen that is consumed in the conversion of substrate. Because of the stoichiometry of the reaction, the effective oxygen consumption rate is half that of the substrate consumption rate because of the catalase side reaction.

The system of differential equations for substrate conversion rate, enzyme inactivation rate and oxygen transfer rate was solved numerically through a step solver (forward Euler method) implemented in Microsoft Excel. The step length for the solver was chosen so that neither of the parameters $\mathrm{S}$ or $\mathrm{O}_{2}$ changed more than $3 \%$ in a single step.

Some of the parameters used in the simulations $\left(k_{c a t}, K_{M, S}\right.$ and $\left.K_{M, O}\right)$ were determined experimentally in a Tube-in-Tube flow reactor ${ }^{47}$ (unpublished data: $480000 \mathrm{~h}^{-1}, 0.03 \mathrm{M}$ and $0.8 \mathrm{mM}$, respectively). To simulate reaction kinetics with high $K_{M, S}$, this parameter was increased to $4 \mathrm{M} . K_{i c}$ and $K_{i u}$ of 1 and $10 \mathrm{mM}$, respectively, were introduced to simulate competitive and uncompetitive inhibition behavior (based on the product concentration). Standard initial concentrations for $S_{0}$ and $E_{0}$ were $0.25 \mathrm{M}$ and $1.45 \mu \mathrm{M}$, respectively. It should be noted that $E_{0}$ was adjusted for each simulated limitation to scale the activity and to allow easy comparison of the different phenomena. The concentration of $S_{0}$ and $E_{0}$ were doubled to simulate high loads of each parameter. 
The parameters describing enzyme kinetics, $k_{d}, k_{d, X}$ and $T T N$ were set to $0.68 \mathrm{~h}^{-1}, 8 \mathrm{~h}^{-1} \mathrm{M}^{-1}$ and 30 000, respectively. Conversion-dependent inactivation behavior was based on the product concentration.

Finally, the rate of oxygen mass transfer was based on a value for $\mathrm{O}_{2}{ }^{*}$ of $0.27 \mathrm{mM}$, calculated through Henry's law based on $21 \% \mathrm{O}_{2}$ (air) in saturation with pure water at $25^{\circ} \mathrm{C}$ and $1 \mathrm{~atm}$. Values of $k_{L} a$ of $20 \mathrm{~h}^{-1}$ and $200 \mathrm{~h}^{-1}$ were applied to simulate systems limited and not limited by oxygen mass transfer, respectively. ${ }^{46}$

GLUCOSE OXIDASE (GOx). Glucose oxidase (GOx) from Aspergillus niger, was supplied by DuPont Industrial Biosciences (Wageningen, The Netherlands) as a freeze-dried powder with a total protein content of $51.25 \%$ (determined by BCA method) and activity of $16.2 \mathrm{U} / \mathrm{mg}$ of lyophilized powder ( 1 Unit $(\mathrm{U})$ decomposes $1 \mu \mathrm{mol}$ of glucose per minute at $\mathrm{pH} 7.5,25{ }^{\circ} \mathrm{C}$, $100 \mathrm{mM}$ glucose). Catalase with $\geq 3000 \mathrm{U} / \mathrm{mg}$ of activity was acquired from Sigma-Aldrich (St. Louis, MO, USA) (1 Unit (U) decomposes $1 \mu \mathrm{mol}$ of $\mathrm{H}_{2} \mathrm{O}_{2}$ per min at $\mathrm{pH} 7.0,25{ }^{\circ} \mathrm{C}, 10 \mathrm{mM}$ $\mathrm{H}_{2} \mathrm{O}_{2}$ ). Analytical grade D-glucose and sodium D-gluconate were obtained from Thermo Fisher Scientific (Waltham, MA, USA); potassium dihydrogen phosphate and dipotassium hydrogen phosphate were acquired from VWR (Radnor, PA, USA) and sodium hydroxide from SigmaAldrich (St. Louis, MO, USA).

Batch reactions were carried in a stirred tank reactor (MiniBio with my-Control from Applikon Biotechnology (Delft, The Netherlands)). The reactor contained a metal sparger, two Rushton turbines, as well as temperature and $\mathrm{pH}$ control. Stirring $(500 \mathrm{rpm})$ and aeration rate (1 volume of air per volume per minute (vvm)) were kept constant. Experiments were performed under the following conditions: operating temperature of $25^{\circ} \mathrm{C}, 200 \mathrm{mM}$ phosphate buffer at $\mathrm{pH} 7.5$, working volume of $150 \mathrm{~mL}$, and catalase concentration of $5 \mathrm{mg} / \mathrm{L}(15860 \mathrm{U} / \mathrm{L})$ to ensure total 
$\mathrm{H}_{2} \mathrm{O}_{2}$ conversion. Glucose concentrations were varied from 10, 20 and $200 \mathrm{mM}$ and $\mathrm{GOx}$ concentrations of 10, 20, 50 and $100 \mathrm{mg} / \mathrm{L}$ (mg of lyophilized powder) were tested. Reactions were carried out until complete oxidation of glucose was achieved and followed by measuring dissolved oxygen in the liquid phase using a Fiber-Optic Oxygen Sensor Probe from PyroScience (GmbH, Aachen, Germany). Samples of $1 \mathrm{~mL}$ were taken at regular intervals (which varied between experiments to fit with the added catalytic activity) and were analyzed by HPLC.

Glucose and gluconic acid in the samples were analyzed by HPLC using the same procedure described by Toftgaard Pedersen and co-workers. ${ }^{48}$

MONOAMINE OXIDASE (MAO). Monoamine oxidase (MAO) from Aspergillus niger for whole-cell biocatalysis was produced through fermentation as reported previously (specific activity not determined). ${ }^{42}$ The substrate, aza-bicyclo-octane $\mathrm{HCl}$ was procured from $\mathrm{AK}$ Scientific (Union City, CA, USA). All other chemicals were purchased from Sigma-Aldrich GmbH (Steinheim, Germany).

Biocatalysis was carried out in baffled shake flasks with a final working volume $20 \mathrm{~mL}$ of reaction mixture (substrate and biocatalyst suspended in $500 \mathrm{mM}$ phosphate buffer, $\mathrm{pH}$ 7.6) incubated at $37^{\circ} \mathrm{C}$ and $150 \mathrm{rpm}$. The whole-cell biocatalyst and substrate concentration as well as sampling frequency and duration were all varied depending on the experiment. Similar experiments were performed with the same batch of cells (to avoid variation between batches).

Substrate and product concentrations in reaction samples were analyzed by GC using a previously published method. ${ }^{42}$

BIODIESEL PRETREATMENT BY IMMOBILIZED LIPASE. Immobilized Candida antarctica lipase B (N435) (specific activity not determined) was kindly donated by Novozymes 
A/S (Bagsværd, Denmark). Degummed rapeseed oil was kindly donated by Emmelev A/S (Otterup, Denmark). The oil had an FFA content of $0.82 \%\left(\mathrm{w} / \mathrm{w}_{\mathrm{oil}}\right)$, with a FFA composition (by weight) of $4.8 \%$ palmitic acid, $1.8 \%$ stearic acid, $63.7 \%$ oleic acid, $18.8 \%$ linoleic acid, $7.9 \%$ linolenic acid and 3.0\% other FFA. The oil was spiked with $95 \%$ oleic acid (Sigma-Aldrich GmbH, Steinheim, Germany) to simulate low-grade oil. Methanol (99.9\%) was purchased from Kemetyl A/S (Køge, Denmark). HPLC calibration standards, methyl oleate ( $\geq 99 \%)$, 1monooleoyl-rac-glycerol $(\geq 99 \%)$ and dioleoylglycerol $(\geq 99 \%$, a mixture of $1.3-$ and $1.2-$ isomers), and HPLC-grade solvents for the analyses, n-Heptane ( $\geq 99 \%)$ and t-butyl methyl ether ( $\geq 99.8 \%$ ), were purchased from Sigma-Aldrich.

Batch experiments were carried out at $45^{\circ} \mathrm{C}$ in $4 \mathrm{~mL}$ capped glass vials, using an HLC MKR13 thermomixer at $1000 \mathrm{rpm}$ (HLC, Bovenden, Germany). Reactions were prepared by weighing catalyst into the vials and subsequently adding $2 \mathrm{~mL}$ rapeseed oil together with the amount of oleic acid required to reach the desired initial FFA concentration for the experiment. The vials were then mixed and preheated to the reaction temperature in the thermoshaker, and an initial sample withdrawn. The reaction was then started by adding methanol. The vials were removed briefly from the thermomixer for sampling. The sample size was $55 \mu \mathrm{L}$, withdrawn using a $100 \mu \mathrm{L}$ glass syringe. Further details of experimental conditions have been published previously. $^{43}$

Details of the analytical procedure has been described previously. ${ }^{43}$

\section{AUTHOR INFORMATION}

\section{Corresponding Author}

*Email (John M. Woodley): jw@kt.dtu.dk 


\section{Author Contributions}

The manuscript was written through contributions of all authors. All authors have given approval to the final version of the manuscript. $\$$ These authors contributed equally.

\section{Funding Sources}

The research for this work has received funding from the European Union (EU) project ROBOX (grant agreement $n^{\circ}$ 635734) under EU's Horizon 2020 Programme Research and Innovation actions H2020-LEIT BIO-2014-1. Any statements herein reflects only the authors' views. The European Union is not liable for any use that may be made of the information contained herein.

The research for the MAO results has received funding from the European Union's Seventh Framework Programme FP7/2007-2013 under grant agreement n 266025 (BIONEXGEN).

\section{Notes}

The authors declare no competing financial interest.

\section{ACKNOWLEDGMENTS}

The research for this work has received funding from the European Union (EU) project ROBOX (grant agreement $n^{\circ}$ 635734) under EU's Horizon 2020 Programme Research and Innovation actions H2020-LEIT BIO-2014-1. Any statements herein reflects only the author's views. The European Union is not liable for any use that may be made of the information contained herein.

The authors would like to thank Professor N. J. Turner (University of Manchester) and his group for providing the plasmids containing MAO. The research for the MAO results has 
received funding from the European Union's Seventh Framework Programme FP7/2007-2013 under grant agreement $\mathrm{n}^{\circ} 266025$ (BIONEXGEN).

\section{REFERENCES}

(1) Sheldon, R. A.; Woodley, J. M. Role of biocatalysis in sustainable chemistry, Chem. Rev. 2018, 118, 801-838.

(2) Pollard, D. J.; Woodley, J. M. Biocatalysis for pharmaceutical intermediates: The future is now, Trends Biotechnol. 2007, 25, 66-73.

(3) Hohne, M.; Bornscheuer, U. T. Biocatalytic routes to optically active amines, ChemCatChem 2009, 1, 42-51.

(4) Nestl, B. M.; Nebel, B. A.; Hauer, B. Recent progress in industrial biocatalysis, Curr. Opin. Chem. Biol. 2011, 15, 187-193.

(5) Turner, N. J. Enantioselective oxidation of C-O and C-N bonds using oxidases, Chem. Rev. 2011, 111, 4073-4087.

(6) Nestl, B. M.; Hammer, S. C.; Nebel, B. A.; Hauer, B. New generation of biocatalysts for organic synthesis, Angew. Chem. Int. Ed. 2014, 53, 3070-3095.

(7) Ghisliere, D.; Turner, N. J. Biocatalytic approaches to the synthesis of enantiomerically pure chiral amines, Top. Catal. 2014, 57, 284-300.

(8) Kohls, H.; Steffen-Munsberg, F.; Hohne, M. Recent achievements in developing the biocatalytic toolbox for chiral amine synthesis, Curr. Opin. Chem. Biol. 2014, 19, 180-192. 
(9) Simon, R. C.; Richter, N.; Busto, E.; Kroutil, W. Recent developments of cascade reactions involving $\omega$-transaminases, ACS Catal. 2014, 4, 129-143.

(10) Wang, J.-B.; Reetz, M. T. Chiral cascades, Nature Chem. 2015, 7, 948-949.

(11) Lalonde, J. Highly engineered biocatalysts for efficient small molecule pharmaceutical synthesis, Curr. Opin. Biotechnol. 2016, 42, 152-158.

(12) France, S. P.; Hepworth, L. J.; Turner, N. J.; Flitsch, S. L. Constructing biocatalytic cascades: In vitro and in vivo approaches to de novo multi-enzyme pathways, ACS Catal. 2014, 4, 129-143.

(13) Schrittwieser, J. H.; Velikogne, S.; Hall, M.; Kroutil, W. Artificial biocatalytic cascades for preparation of organic molecules, Chem. Rev. 2018, 118, 270-348.

(14) Sheldon, R. A.; Brady, D. The limits to biocatalysis: pushing the envelope. Chem. Commun. 2018, 54, 6088-6104.

(15) Woodley, J. M. Bioprocess intensification for the effective production of chemical products, Comput. Chem. Eng. 2017, 105, 297-307.

(16) Strohmeier, G. A.; Pichler, H.; May, O.; Gruber-Khadjawi, M. Application of designed enzymes in organic synthesis, Chem. Rev. 2011, 111, 4141-4164.

(17) Bornscheuer, U. T.; Huisman, G. W.; Kazlauskas, R. J.; Lutz, S.; Moore, J. C.; Robins, K. Engineering the third wave of biocatalysis, Nature 2012, 485, 185-194. 
(18) Liszla, M. J.; Clark, M. E.; Schneider, E.; Clark, D. S. Nature versus nurture: Developing enzymes that function under extreme conditions, Annu. Rev. Chem. Biomol. Eng. 2012, 3, 77-102.

(19) Reetz, M. T. What are the limitations of enzymes in synthetic organic chemistry, Chem. Rec. 2016, 16, 2449-2459.

(20) Savile, C. K.; Janey, J. M.; Mundroff, E. C.; Moore, J. C.; Tam, S.; Jarvis, W. R; Colbeck, J. C.; Krebber, A.; Fleitz, F. J.; Brands, J.; Devine, P. N.; Huisman, G. W.; Hughes, G. J. Biocatalytic asymmetric synthesis of chiral amines from ketones applied to sitagliptin manufacture, Science 2010, 329, 305-309.

(21) Bloom, J. D.; Labthavikul, S. T.; Otey, C. R.; Arnold, F. H. Protein stability promotes evolvability, Proc. Natl. Acad. Sci. U.S.A. 2006, 103, 5869-5874.

(22) Cahn, J. K. B.; Werlang, C. A.; Baumschlager, A.; Brinkmann-Chen, S.; Mayo, S. L.; Arnold, F. H. A general tool for engineering the NAD/NADP cofactor preference of oxidoreductases, ACS Synth. Biol. 2017, 6, 326-333.

(23) Turner, N. J. Directed evolution drives the next generation of biocatalysts, Nat. Chem. Biol. 2009, 5, 567-573.

(24) Truppo, M. D. Biocatalysis in the pharmaceutical industry: The need for speed, ACS Med. Chem. Lett. 2017, 8, 476-480.

(25) Wohlgemuth, R. Interfacing biocatalysis and organic synthesis, J. Chem. Technol. Biotechnol. 2007, 82, 1055-1062. 
(26) Cornish-Bowden, A. in Fundamentals of Enzyme Kinetics; Portland Press 1td.: London, 2004.

(27) Gardossi, L.; Poulsen, P. B.; Ballesteros, A.; Hult, K.; Svedas, V. K.; Vasić-Racki, D.; Carrea, G.; Magnusson, A.; Schmid, A.; Wohlgemuth, R.; Halling, P. J. Guidelines for reporting of biocatalytic reactions, Trends Biotechnol. 2010, 28, 171-180.

(28) Blackmond, D. G. Reaction progress kinetic analysis: A powerful methodology for mechanistic studies of complex catalytic reactions, Angew. Chem. Int. Ed. Engl. 2005, 44, $4302-4320$.

(29) Duggleby, R. G. Quantitative analysis of the time courses of enzyme-catalyzed reactions, Methods 2001, 24, 168-174.

(30) Selwyn, M. J. A simple test for inactivation of an enzyme during assay, Biochim. Biophys. Acta 1965, 105, 193-195.

(31) Meissner, M. P.; Nordblad, M.; Woodley, J. M. Online measurement of oxygendependent enzyme reaction kinetics, ChemBioChem 2017, 19, 106-113.

(32) Toftgaard Pedersen, A.; Birmingham, W. R.; Rehn, G.; Charnock, S. J.; Turner, N. J.; Woodley, J. M. Process requirements of galactose oxidase catalyzed oxidation of alcohols, Org. Process Res. Dev. 2015, 19, 1580-1589.

(33) Stocks, S. M. in Microbial Production of Food Ingredients, Enzymes and Nutraceuticals; Woodhead: Cambridge, 2013; pp. 144-172.

(34) Gasparini, G.; Archer, I.; Jones, E.; Ashe, R. Scaling up biocatalysis reactions in flow reactors, Org. Process Res. Dev. 2012, 16, 1013-1016. 
(35) Bommarius, A. S.; Paye, M. F. Stabilizing biocatalysts, Chem. Soc. Rev. 2013, 42, 65346565.

(36) Henley, J. P.; Sadana, A. Categorization of enzyme deactivations using a series-type mechanism, Enzyme Microb. Technol. 1985, 7, 50-60.

(37) Slavica, A.; Dib, I.; Nidetzky, B. Single-site oxidation, cysteine 108 to cysteine sulfinic acid, in D-amino acid oxidase from Trigonopsis variabilis and its structural and functional, Appl. Environ. Microbiol. 2005, 71, 8061-8068.

(38) Moo-Young, M.; Kobayashi, M. Effectiveness factors for immobilized-enzyme reactions, Can. J. Chem. Eng. 1972, 50, 162-167.

(39) Wang, D. I. C.; Cooney, C. L.; Demain, A. L.; Dunnill, P.; Humphrey, A. E.; Lilly, M. D. in Fermentation and Enzyme Technology; John Wiley \& Sons: New York, NY, 1979; Vol. 18, pp. 311-338.

(40) Liese, A.; Hilterhaus, L. Evaluation of immobilized enzymes for industrial applications. Chem. Soc. Rev. 2013, 42, 6236-6249.

(41) Verhoff, F. H.; Goldstein, W. E. Diffusion resistance and enzyme activity decay in a pellet, Biotechnol. Bioeng. 1982, 24, 703-723.

(42) Ramesh, H.; Woodley, J. M. Process characterization of a monoamine oxidase, J. Mol. Catal. B Enzym. 2014, 106, 124-131.

(43) Nordblad, M.; Pedersen A. K.; Rancke-Madsen, A.; Woodley, J. M. Enzymatic pretreatment of low-grade oils for biodiesel production, Biotechnol. Bioeng. 2016, 113, 754-760. 
(44) Ramesh, H.; Nordblad, M.; Whitall, J.; Woodley, J. M. in Practical Methods for Biocatalysis and Biotransformations 3; Wiley Online Library, 2016; pp. 1-30.

(45) Tufvesson, P.; Lima-Ramos, J.; Nordblad, M.; Woodley, J. M. Guidelines and cost analysis for catalyst production in biocatalytic processes, Org. Process Res. Dev. 2011, 15, 266-274.

(46) Villadsen, J. in Fundamental Bioengineering; Wiley-Blackwell: Hoboken, NJ, 2016.

(47) Ringborg, R. H.; Toftgaard Pedersen, A.; Woodley, J. M. Automated determination of oxygen-dependent enzyme kinetics in a tube-in-tube flow reactor, ChemCatChem 2017, 9 , 3285-3288.

(48) Toftgaard Pedersen, A.; de Carvalho, T. M.; Sutherland, E.; Rehn, G.; Ashe, R.; Woodley, J. M. Characterization of a continuous agitated cell reactor for oxygen dependent biocatalysis, Biotechnol. Bioeng. 2017, 114, 1222-1230. 